\title{
Incorporating Change-Point Detection Updates of Frequency-Magnitude Distributions Within the Taiwan Earthquake Catalog
}

\author{
Kuei-Pao Chen ${ }^{1, *}$, Yi-Ben Tsai ${ }^{2}$, Daniel Amorese ${ }^{3}$, and Wen-Yen Chang ${ }^{4}$ \\ ${ }^{1}$ Hsin Sheng College of Medical Care and Management, Taoyuan, Taiwan, ROC \\ ${ }^{2}$ Geosciences Department, Pacific Gas and Electric Company, San Francisco, USA \\ ${ }^{3}$ University of Caen, Caen, France \\ ${ }^{4}$ Department of Natural Sciences of National Science Council, Taipei, Taiwan, ROC
}

Received 3 May 2010, accepted 17 September 2010

\begin{abstract}
Threshold magnitude $\left(M_{0}\right)$ is an important factor in determining the magnitude of completeness in calculating seismic $b$ values. Seismic $b$ values can assist in determining the likelihood of earthquake events; therefore, seismic $b$ values should be re-determined repeatedly with ever increasing precision. In this study, we use a median-based analysis of the segment slope (MBASS) to detect change points in Gutenberg-Richter frequency-magnitude distributions (FMDs) to determine $M_{0}$. Results give the $b$ value for $M_{0}-0.5$ to be smaller than the $b$-value for $M_{0}+0.5$, and the difference in $b$ values between $M_{0}$ and $M_{0}-0.5$ is larger than the difference in $b$ values between $M_{0}$ and $M_{0}+0.5$. Therefore, $b$ values resulting from $M_{0}+0.5$ should therefore be more accurate than $b$ values from $M_{0}-0.5$ when calculating $b$ values using the threshold magnitude $\left(M_{0}\right)$. This is especially true when earthquake events are few and $M_{0}$ is large such as for the time periods 1900 - 1935, and 1936 - 1972 as noted in of Taiwan's earthquake catalog.
\end{abstract}

Key words: Threshold magnitude, Median-based analysis of the segment slope, Change-point, Frequency-magnitude distribution

Citation: Chen, K. P., Y. B. Tsai, D. Amorese, and W. Y. Chang, 2011: Incorporating change-point detection updates of frequency-magnitude distributions within the Taiwan earthquake catalog. Terr. Atmos. Ocean. Sci., 22, 261-269, doi: 10.3319/TAO.2010.09.17.01(T)

\section{INTRODUCTION}

Since the installation of Taiwan's first seismograph in 1897, Taiwan's earthquake catalog has been relatively complete. However, changes in seismographic characteristics, network coverage, and observational practice, as well as definitional and procedural changes in magnitude determination led to inconsistent measures of earthquake magnitude. Wang (1992) also reviewed in details the magnitude scales and their relationships for earthquakes in Taiwan. Chen and Tsai (2008) recompiled the different definitions of magnitude for five different time periods into a uniform standard: moment magnitude $\left(M_{W}\right)$ (see Fig. 1a). To the homogenized $M_{W}$ of Fig. 1a, we add supplementary data found in the literature for the period from 1900 to 1935 (see Fig. 1b) with the results which enriches the content with magnitude $M_{W} \geq 5.5$ during this period. Therefore, this article is based on the dataset displayed in Fig. $1 \mathrm{~b}$.

\footnotetext{
* Corresponding author

E-mail: chenkueipao@yahoo.com.tw
}

We then use the size distribution of the earthquake Gutenberg-Richter frequency-magnitude relationship (Ishimoto and Iida 1939; Gutenberg and Richter 1944) to evaluate $b$ values, in most cases the fundamental relation is expressed as:

$\log _{10} N(M)=a-b M$

where $a$ is proportional to the seismicity rate; $b$ describes the size distribution of events; $M$ is the magnitude; and $N(M)$ is the cumulative number of earthquakes with magnitude equal to or greater than $M$. The $b$ value is an essential and important factor in seismic hazard analysis and demands ever increasing accuracy as advances in scholarship permit. Therefore, we must accurately calculate $b$ values by first determining threshold magnitude $\left(M_{0}\right)$, and then following maximum likelihood procedures to find $b$ values. For example, Enescu et al. (2009) used the $c$ value of the OmoriUtsu law to detect the magnitude of completeness for a series of aftershock sequences in Japan and then determine 

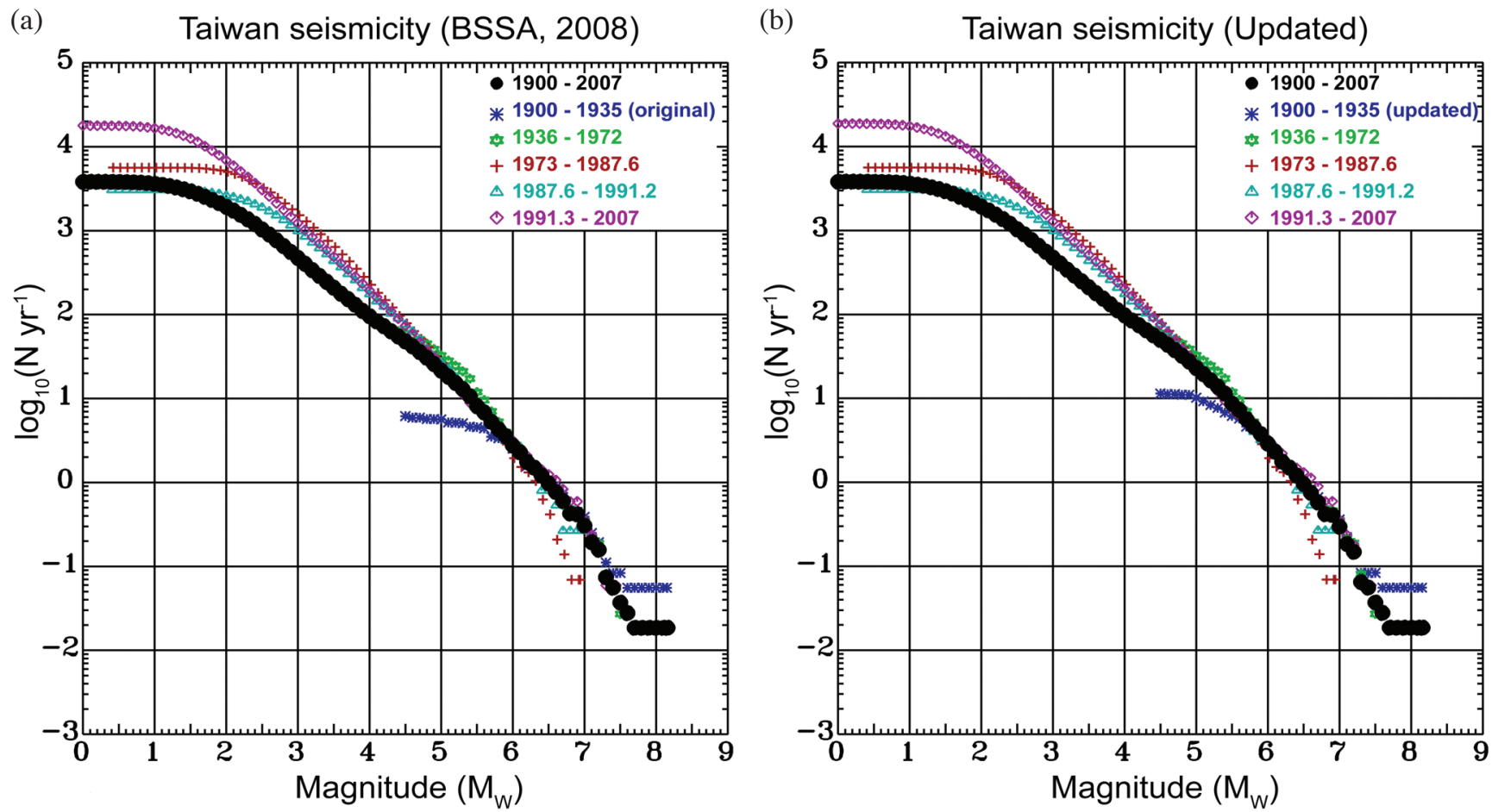

Fig. 1. (a) Plots of the logarithm of cumulative number of earthquakes per year versus the new $M_{W}$ magnitude for Taiwan earthquakes over different time periods since 1900. (b) Plots of the logarithm of cumulative number of earthquakes per year versus the new $M_{W}$ magnitude for Taiwan earthquakes over different time periods since 1900, with additional data for 1900 - 1935.

the threshold magnitude $\left(M_{0}\right)$. They then used a maximum likelihood procedure (Aki 1965; Utsu 1965) to calculate $b$ values. Wiemer et al. (2002) developed a "window" of 100 events, with an $M_{0}$ calculated for every 50 events, and then applied a maximum likelihood method (Aki 1965) to calculate $b$ values. In addition, various methods have been suggested to measure $b$ values and its confidence limits (Aki 1965; Utsu 1965, 1992; Shi and Bolt 1982; Bender 1983; Wang 1988; Frohlich and Davis 1993; Kagan 1999; Wiemer 2002).

\section{METHOD}

Some breakpoints certainly exist in the earthquake Gutenberg-Richter frequency-magnitude distribution (see Fig. 2). Such breakpoints are useful in determining the threshold magnitude $\left(M_{0}\right)$ for calculating $b$ values and correct determination of $b$ values is likewise dependent upon accurate analysis of threshold magnitudes $\left(M_{0}\right)$.

In this study, we use a nonparametric statistical method called median-based analysis of the segment slope (MBASS) (Amorese 2007). This method can help to detect changepoints of lower magnitudes to find the threshold magnitude $\left(M_{0}\right)$, and also detect breakpoints toward higher magnitudes. The method is briefly described as below:

If $\mathrm{M}_{1}$ and $\mathrm{M}_{2}$ are the magnitudes of two consecutive points of the FMD, respectively, the segment slope for $M=$
$M_{2}$ was as approximated by :

$s\left(M_{2}\right)=\frac{\log \left[N\left(M_{1}\right)\right]-\log \left[N\left(M_{2}\right)\right]}{M_{1}-M_{2}}$

The segment slope was computed for each magnitude increment based upon an analysis keyed to any magnitude value corresponding to a significant and stable change in the median of the segment slope of the FMD (the method is well described by Amorese 2007).

\section{DATA AND PROCESSING}

In this study, datasets are based on Chen and Tsai's (2008) recompilation of different definitions of magnitude for five different periods into a uniform moment magnitude $\left(M_{W}\right)$ (see Fig. 1a). For this study the catalog has been updated to include earthquakes occurred up to 2007 , as well as add supplementary data found in the literature for the time period 1900 - 1935 (see Fig. 1b). Data, in this study, is based on Fig. 1b. Descriptions of the five different periods in Taiwan's earthquake catalog are given below:

(1) 1900 - 1935 dataset: a subset of the Taiwan earthquake catalog recompiled from magnitude $M_{H}$ into $M_{W}$.

(2) 1936 - 1972 dataset: a subset of the Taiwan earthquake catalog recompiled from magnitude $M_{H}$ into $M_{W}$. 
(3) 1973 - 1987.6 dataset: a subset of the Taiwan earthquake catalog recompiled from magnitude $M_{D(A)}$ into $M_{W}$.

(4) 1987.6 - 1991.2 dataset: a subset of the Taiwan earthquake catalog recompiled from magnitude $M_{D(D)}$ into $M_{W}$.

(5) 1991.3 - 2007 dataset: a subset of the Taiwan earthquake catalog recompiled from magnitude $M_{L}$ into $M_{W}$.
After adding additional data to the original dataset for the period 1900 to 1935 , we need to determine the difference in $M_{0}$ for the two datasets. There were too few earthquakes in this time period to use MBASS to determine $M_{0}$ in the original dataset. Therefore, we evaluate $M_{0}$ by plotting the cumulative Gutenberg-Richter frequency-magnitude distribution as shown in Fig. 3, then compare the results

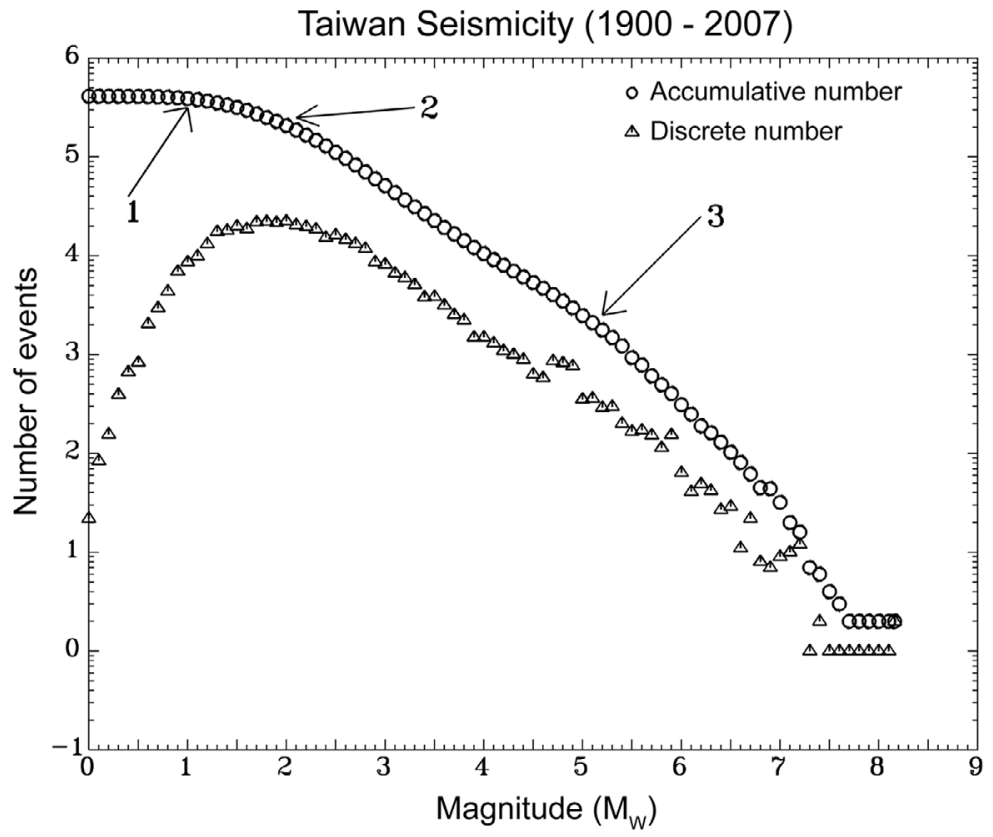

Fig. 2. Examples of Gutenberg-Richter frequency-magnitude distributions from a studied data set (1900 - 2007). The numbered arrows mark apparent breakpoints of the cumulative distribution. Typically, breakpoint 2 has $M_{0}$ magnitude.

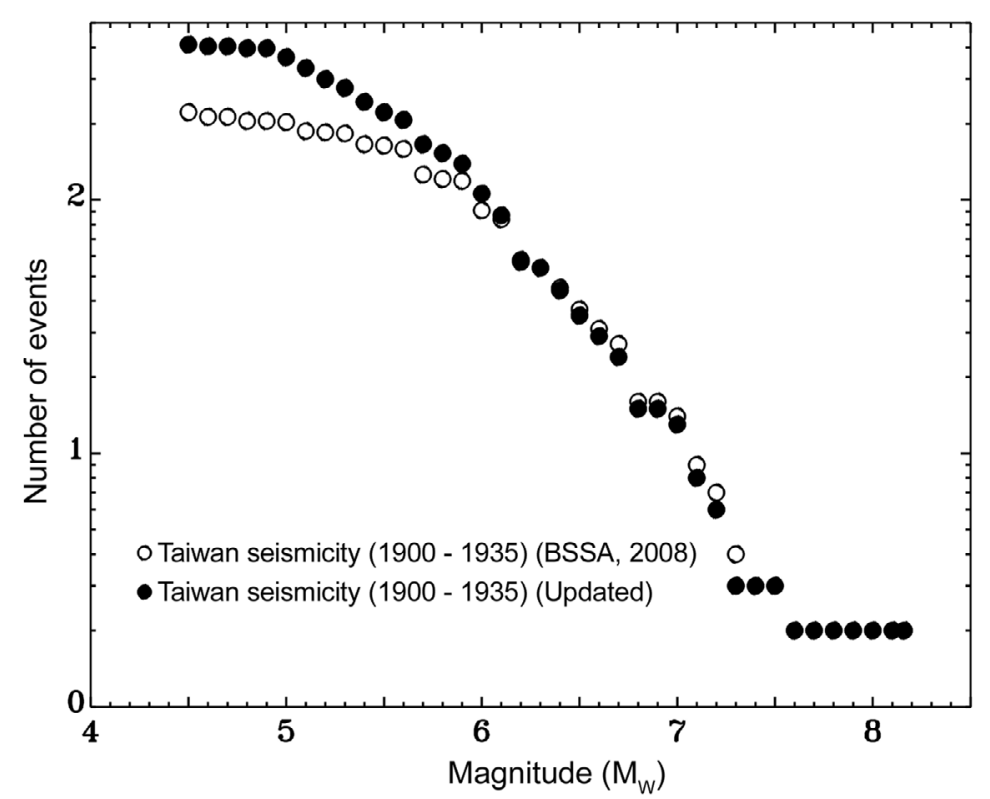

Fig. 3. Gutenberg-Richter frequency-magnitude distributions for the period 1900 - 1935 (BSSA, 2008) dataset (white circles) and with added data (black circles) of cumulative number. By comparing with Fig. 2, there is a distinct slope change at $M_{W} 6.0$ (white circle) and $M_{W} 5.5$ (black circle) along the cumulative Gutenberg-Richter frequency-magnitude distribution; therefore, we determine the $M_{0}$ to be 6.0 and 5.5 respectively. 
with Fig. 2. Figure 3 shows cumulative Gutenberg-Richter frequency-magnitude distribution to have a distinct slope change at magnitude $M_{W} 6.0$. We, therefore, determine $M_{0}$ (original dataset) to be 6.0. After the addition of new data into the original dataset, the number of earthquakes was still too few to use MBASS to determine $M_{0}$. Consequently, we also used the cumulative Gutenberg-Richter frequencymagnitude distribution to determine $M_{0}$ for this data, too (see Fig. 3). Figure 3 shows cumulative Gutenberg-Richter frequency-magnitude distribution having a slope change at magnitude $M_{W} 5.5$. We, therefore, determined the $M_{0}$ (augmented dataset) to be 5.5. This analysis shows that the addition of supplementary data enriched threshold magnitude $\left(M_{0}\right)$ from 6.0 to 5.5 .

For the time periods: 1936 - 1972, 1973 - 1987.6, 1987.6 - 1991.2, and 1991.3 - 2007, we have adequate data to use MBASS to determine $M_{0}$ (see Figs. 4, 5, 6, and 7). The determined $M_{0}$ values are relative, individually, to the main discontinuities as shown in Figs. 4b, 5b, 6b, and 7b, respectively. The change points at high magnitudes are individually relative to auxiliary discontinuity as shown in Figs. 4c, 5c, 6c, and 7c, respectively. In this study, we are generally concerned with the main discontinuity for each period. We list $M_{0}, a$, and $b$ values for the different periods in Table 1.
After applying the MBASS method, we obtain the $b$ value of $M_{0}-0.5$ which is smaller than the $b$ value of $M_{0}+$ 0.5 , with the difference in $b$ value between $M_{0}$ and $M_{0}-0.5$ being larger than the difference in $b$ value between $M_{0}$ and $M_{0}+0.5 . b$ values resulting from $M_{0}+0.5$ should therefore be more accurate than $b$ values resulting from $M_{0}-0.5$ when calculating $b$ values using threshold magnitude $\left(M_{0}\right)$. This is especially true when the amount of earthquakes is few and threshold magnitude is large, as in our database during the time periods 1900 - 1935, and 1936 - 1972. This suggests that using $M_{0}+0.5$ to calculate the $b$ value will cause less error than $M_{0}-0.5$ for $b$ values calculated by $M_{0}$.

\section{RESULTS AND INTERPRETATION}

We apply the MBASS method to determine breakpoints with the resultant values in $M_{0}$ corresponding to the main discontinuity magnitudes. The only important discrepancy is for the time period 1900 - 1935, in which earthquake numbers are too few to use MBASS effectively to determine $M_{0}$. Here, we determine $M_{0}$ by comparing the cumulative Gutenberg-Richter frequency-magnitude distributions (see Fig. 3) with Fig. 2.

MBASS is an effective tool in determining the threshold magnitude $\left(M_{0}\right)$ (Figs. 4, 5, 6, and 7) of the Gutenberg-
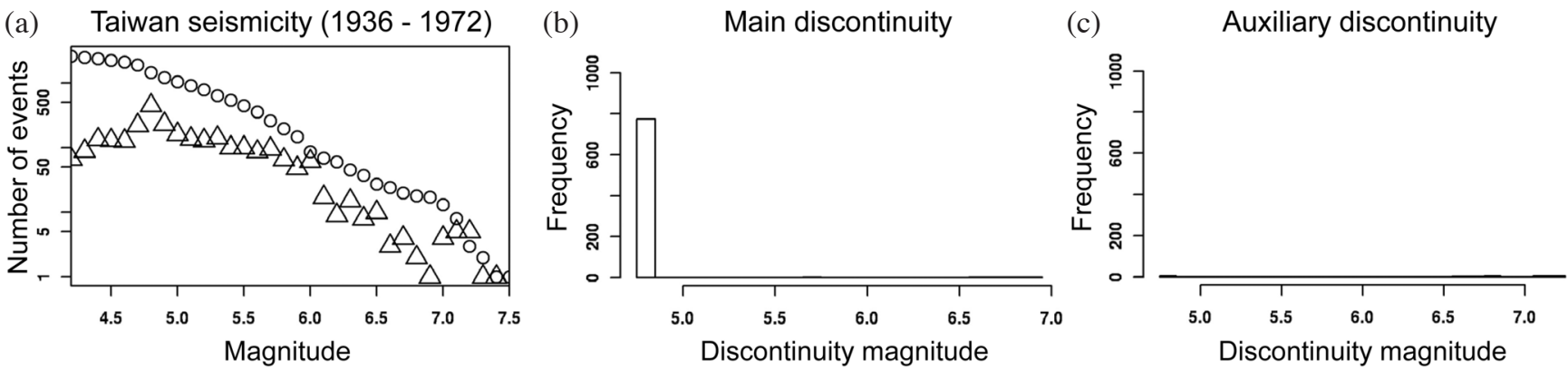

Fig. 4. Gutenberg-Richter frequency-magnitude distributions and histograms of the magnitude discontinuities for the period $1936-1972$. (a) $\mathrm{Cu}-$ mulative (circles) and incremental (triangles) FMDs. (b) Histogram showing the distribution of the Main MBASS magnitude discontinuity ( $M_{0}$ ) obtained from 1000 bootstrap replications. (c) Same as (b) but for the auxiliary discontinuity.

(a) Taiwan seismicity $(1973-1987.6)$

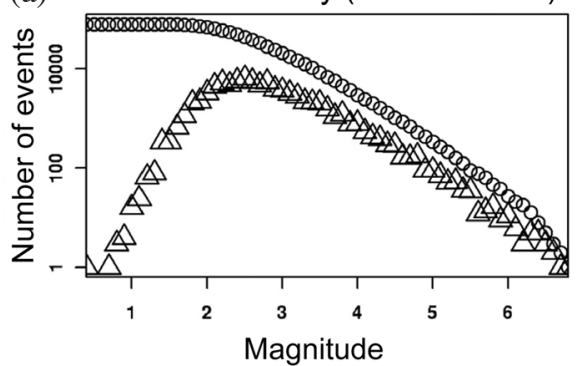

(b)

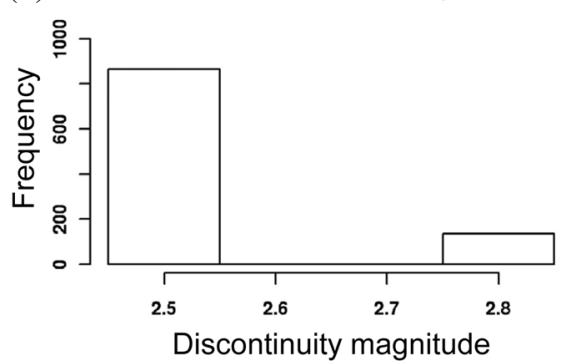

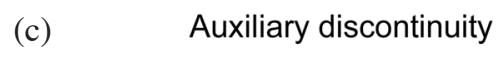

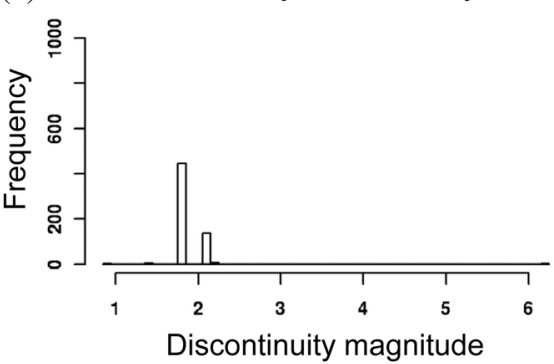

Fig. 5. Gutenberg-Richter frequency-magnitude distributions and histograms of the magnitude discontinuities for the period 1973 - 1987.6. (a) Cumulative (circles) and incremental (triangles) FMDs. (b) Histogram showing the distribution of the Main MBASS magnitude discontinuity $\left(M_{0}\right)$ obtained from 1000 bootstrap replications. (c) Same as (b) but for the auxiliary discontinuity. 

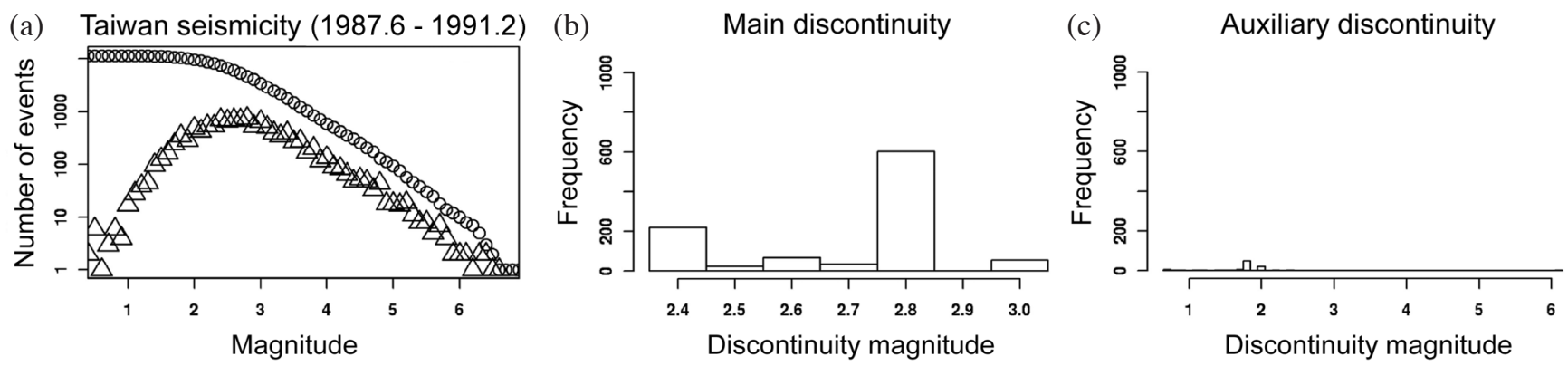

Fig. 6. Gutenberg-Richter frequency-magnitude distributions and histograms of the magnitude discontinuities for the period 1987.6 - 1991.2. (a) Cumulative (circles) and incremental (triangles) FMDs. (b) Histogram showing the distribution of the Main MBASS magnitude discontinuity ( $\left.M_{0}\right)$ obtained from 1000 bootstrap replications. (c) Same as (b) but for the auxiliary discontinuity.
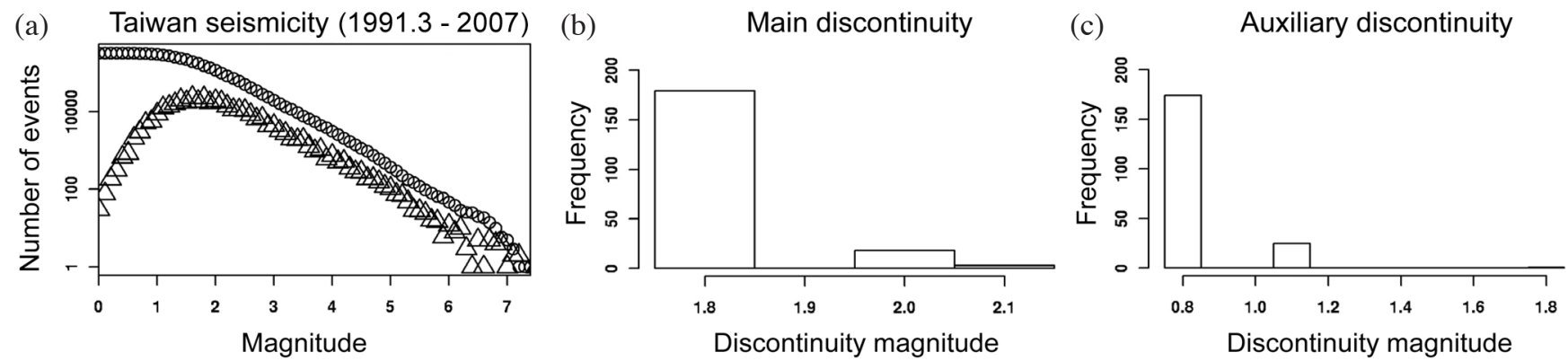

Fig. 7. Gutenberg-Richter frequency-magnitude distributions and histograms of the magnitude discontinuities for the period 1991.3 - 2007. (a) Cumulative (circles) and incremental (triangles) FMDs. (b) Histogram showing the distribution of the Main MBASS magnitude discontinuity ( $M_{0}$ ) obtained from 1000 bootstrap replications. (c) Same as (b) but for the auxiliary discontinuity.

Richter frequency-magnitude distribution for calculating accurate $b$ values. The different $b$ values by $M_{0}$ (for respective time periods) are given in Figs. 8 - 12. MBASS is also useful in the detection of additional discontinuities in the Gutenberg-Richter frequency-magnitude distributions. In this study, even if respective cumulative Gutenberg-Richter frequency-magnitude distributions show apparent \#3 type breakpoints (for breakpoint types see Fig. 2), datasets for the time periods 1936 - 1972, 1973 - 1987.6, 1987.6 - 199.2, and $1991.3-2007$, did not show significant discontinuities when their incremental distributions were investigated using MBASS procedures (Figs. 4c, 5c, 6c, and 7c). These results agree with that found in Amorèse (2007).

We obtain different $b$ values for different $M_{0}$ in the same time periods which are listed in Table 2. From Table 2, it is evident that the $b$ value of $M_{0}-0.5$ is smaller than the $b$ value of $M_{0}+0.5$, and the difference in $b$ value between $M_{0}$ and $M_{0}-0.5$ is larger than the difference in $b$ value between $M_{0}$ and $M_{0}+0.5 . b$ values resulting from $M_{0}+0.5$ should therefore be more accurate than $b$ values from $M_{0}-0.5$ when calculating $b$ values using threshold magnitude $\left(M_{0}\right)$. This is especially true when earthquake numbers for the period are few and the threshold magnitude is large such as between $1900-1935$, and $1936-1972$. This suggests that if we use $M_{0}+0.5$ to calculate $b$ values less error will result than using $M_{0}-0.5$ in $b$ value calculations.
Table 1 . The threshold magnitude $\left(M_{0}\right), a$, and $b$ values of different periods found in the catalog of Taiwan earthquakes.

\begin{tabular}{l|ccc}
\hline & $\boldsymbol{M}_{\boldsymbol{0}}$ & $\boldsymbol{a}$ & $\boldsymbol{b}$ \\
\hline $1900-1935$ & 5.5 & 5.76 & 0.89 \\
$1936-1972$ & 4.8 & 6.77 & 1.04 \\
$1973-1987.6$ & 2.5 & 5.97 & 0.91 \\
$1987.6-1991.2$ & 2.8 & 5.56 & 0.83 \\
$1991.3-2007$ & 1.8 & 5.64 & 0.85 \\
\hline
\end{tabular}

\section{CONCLUSIONS}

A nonparametric statistical method called medianbased analysis of segment slope was applied successfully to Taiwan's earthquake catalog (1900 to 2007) to detect change points in the Gutenberg-Richter frequency-magnitude distributions. This approach is important for determining threshold magnitude $\left(M_{0}\right)$ for use in the application of the Gutenberg-Richter frequency-magnitude relationship to calculate reliable $b$ values. $b$ value is an important factor in seismic hazard analysis, according to Wyss and Stefansson (2006) future mainshocks can be expected along zones characterized by low $b$ values. Therefore, we could obtain a 

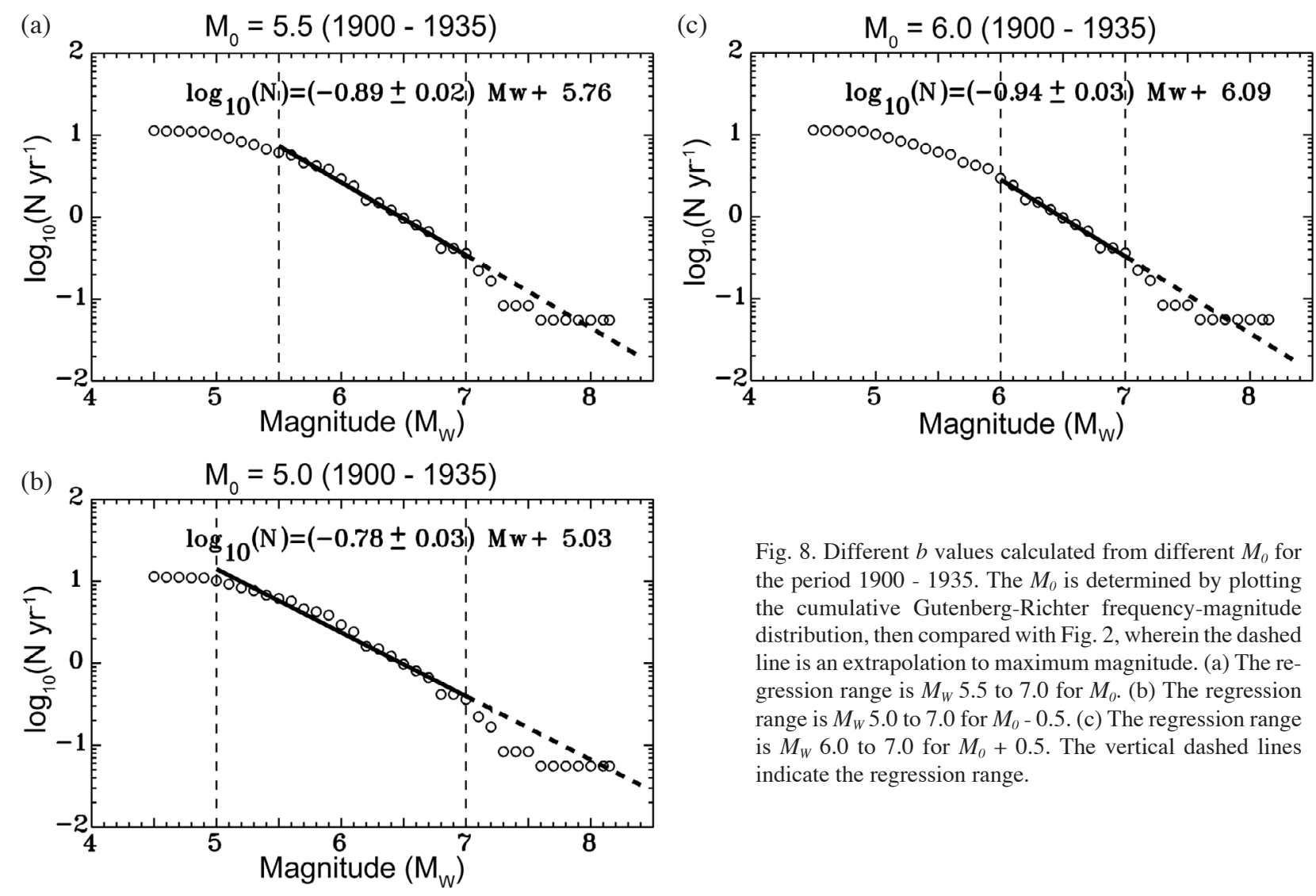

Fig. 8. Different $b$ values calculated from different $M_{0}$ for the period $1900-1935$. The $M_{0}$ is determined by plotting the cumulative Gutenberg-Richter frequency-magnitude distribution, then compared with Fig. 2, wherein the dashed line is an extrapolation to maximum magnitude. (a) The regression range is $M_{W} 5.5$ to 7.0 for $M_{0}$. (b) The regression range is $M_{W} 5.0$ to 7.0 for $M_{0}-0.5$. (c) The regression range is $M_{W} 6.0$ to 7.0 for $M_{0}+0.5$. The vertical dashed lines indicate the regression range.
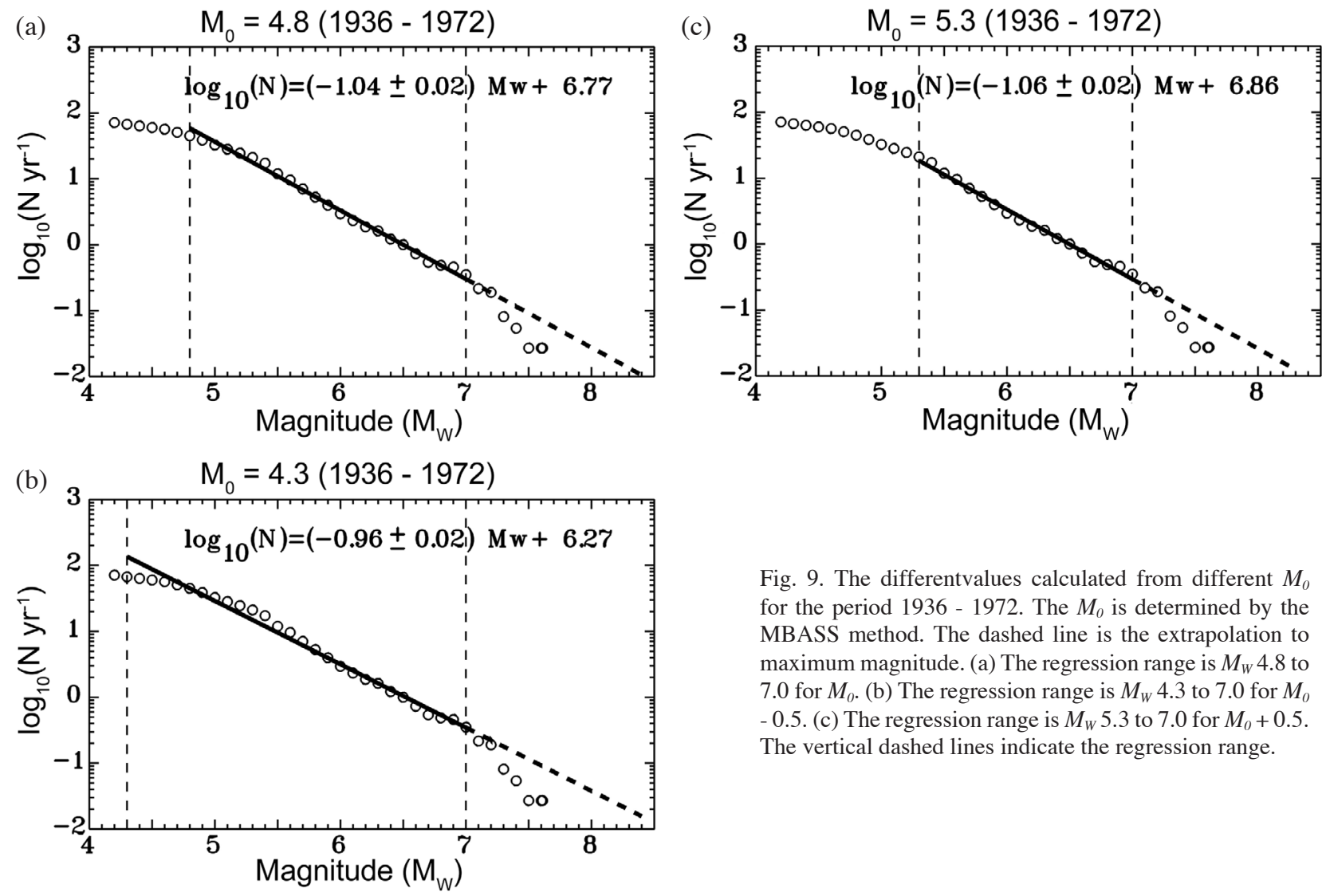

Fig. 9. The differentvalues calculated from different $M_{0}$ for the period $1936-1972$. The $M_{0}$ is determined by the MBASS method. The dashed line is the extrapolation to maximum magnitude. (a) The regression range is $M_{W} 4.8$ to 7.0 for $M_{0}$. (b) The regression range is $M_{W} 4.3$ to 7.0 for $M_{0}$ - 0.5. (c) The regression range is $M_{W} 5.3$ to 7.0 for $M_{0}+0.5$. The vertical dashed lines indicate the regression range. 

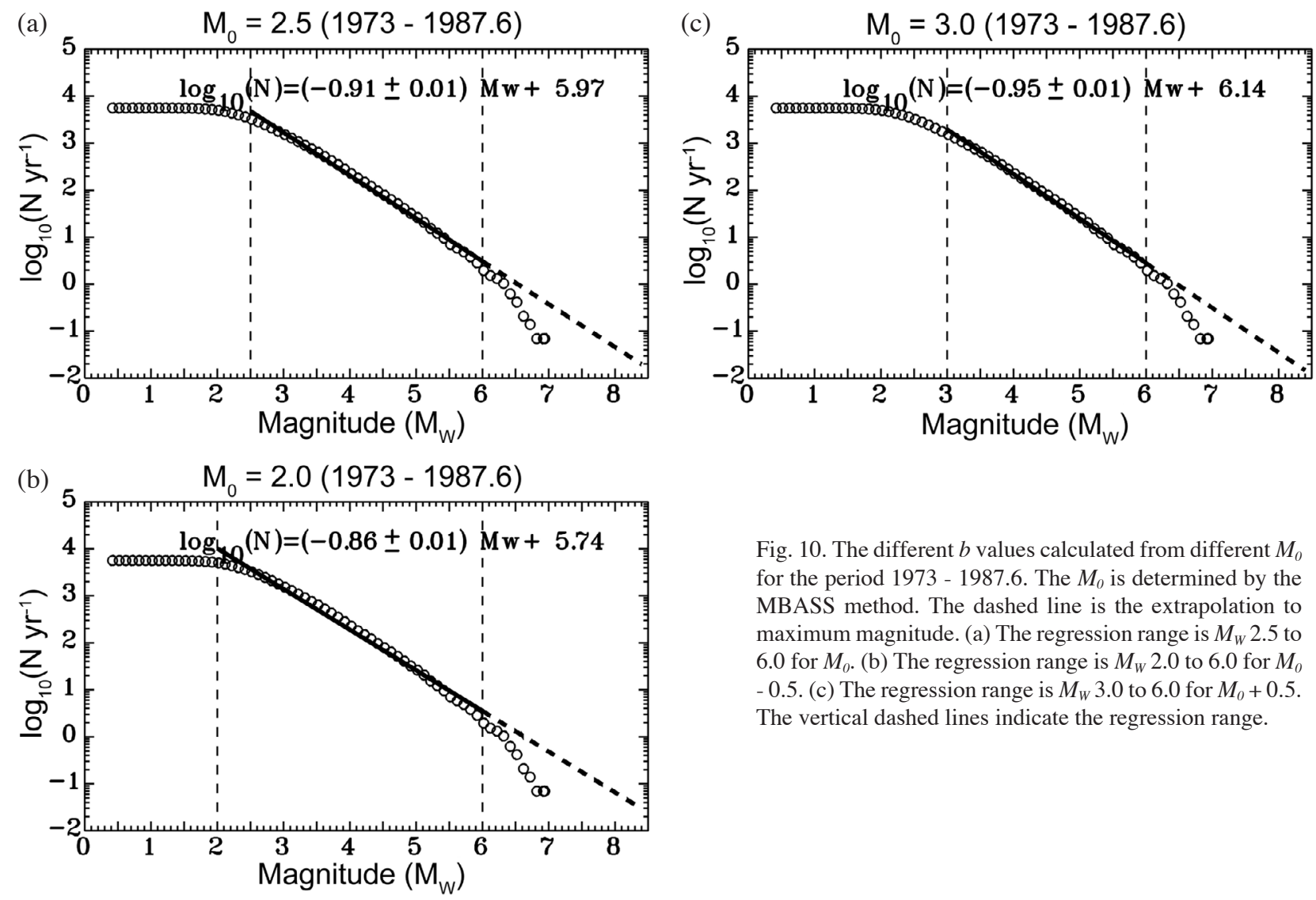

Fig. 10. The different $b$ values calculated from different $M_{0}$ for the period 1973 - 1987.6. The $M_{0}$ is determined by the MBASS method. The dashed line is the extrapolation to maximum magnitude. (a) The regression range is $M_{W} 2.5$ to 6.0 for $M_{0}$. (b) The regression range is $M_{W} 2.0$ to 6.0 for $M_{0}$ - 0.5. (c) The regression range is $M_{W} 3.0$ to 6.0 for $M_{0}+0.5$. The vertical dashed lines indicate the regression range.
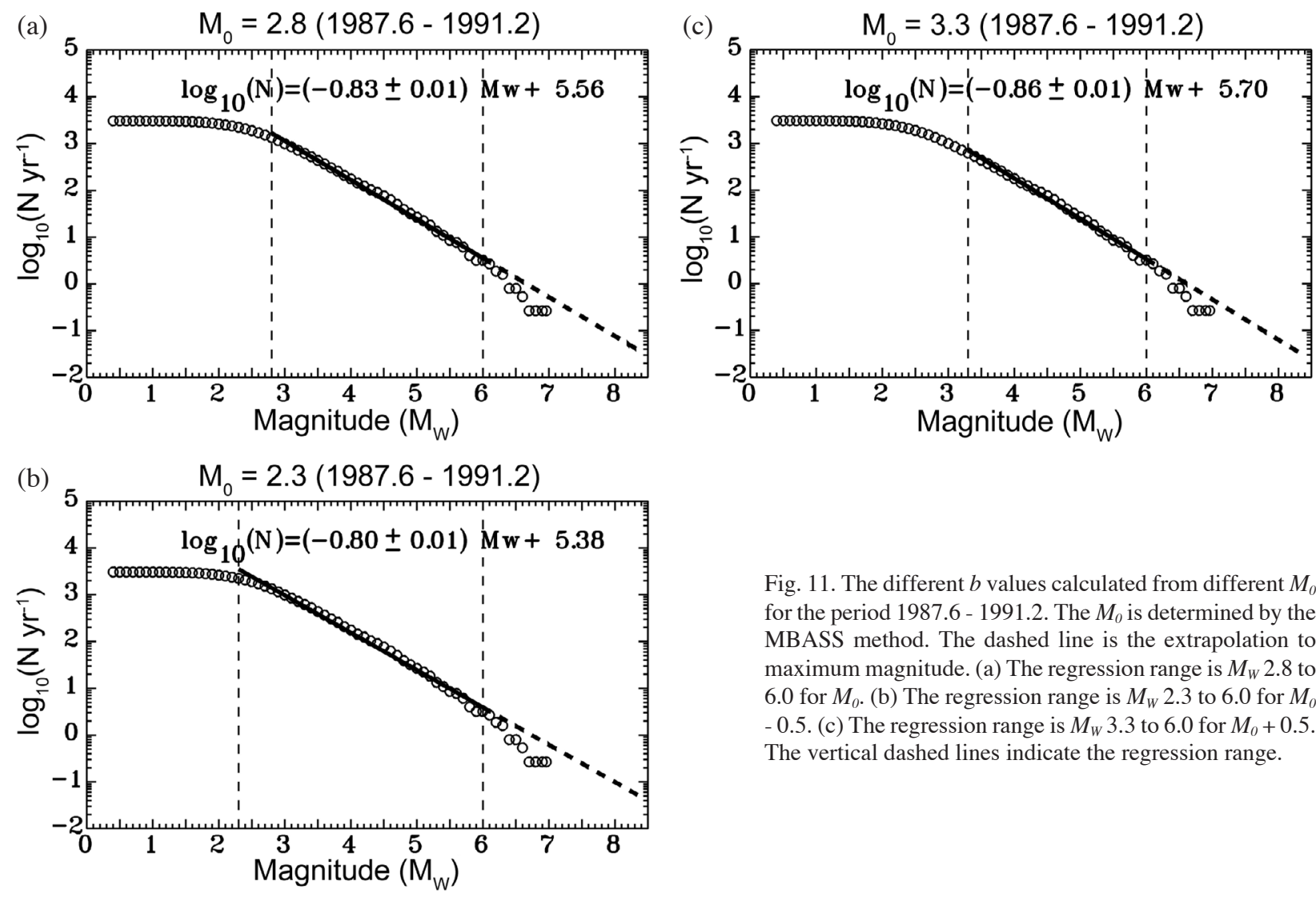

Fig. 11. The different $b$ values calculated from different $M_{0}$ for the period 1987.6 - 1991.2. The $M_{0}$ is determined by the MBASS method. The dashed line is the extrapolation to maximum magnitude. (a) The regression range is $M_{W} 2.8$ to 6.0 for $M_{0}$. (b) The regression range is $M_{W} 2.3$ to 6.0 for $M_{0}$ - 0.5. (c) The regression range is $M_{W} 3.3$ to 6.0 for $M_{0}+0.5$. The vertical dashed lines indicate the regression range. 

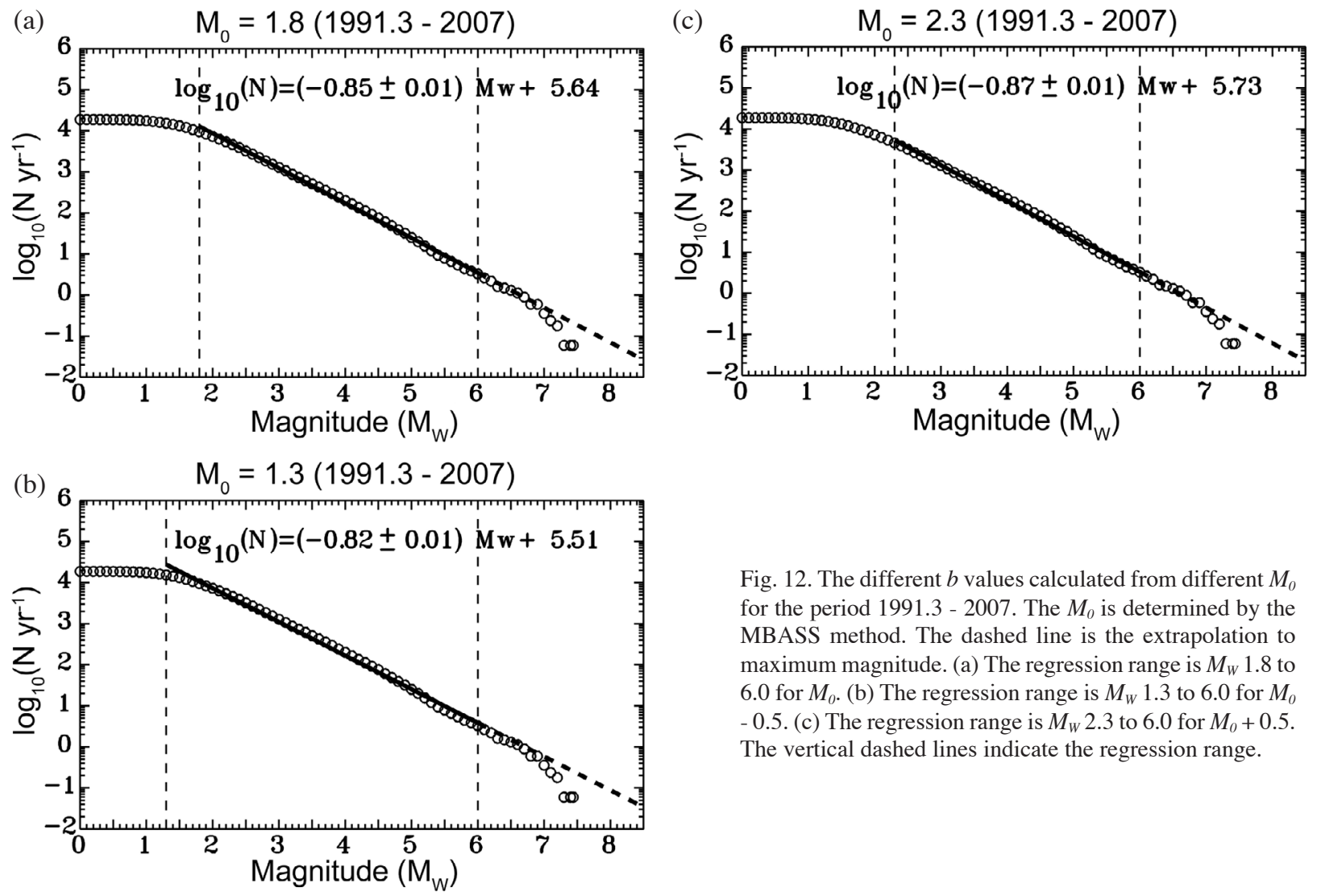

Fig. 12. The different $b$ values calculated from different $M_{0}$ for the period $1991.3-2007$. The $M_{0}$ is determined by the MBASS method. The dashed line is the extrapolation to maximum magnitude. (a) The regression range is $M_{W} 1.8$ to 6.0 for $M_{0}$. (b) The regression range is $M_{W} 1.3$ to 6.0 for $M_{0}$ - 0.5. (c) The regression range is $M_{W} 2.3$ to 6.0 for $M_{0}+0.5$. The vertical dashed lines indicate the regression range.

Table 2. The $a, b$ variation of different $M_{0}$ values of different periods found in the catalog of Taiwan earthquakes.

\begin{tabular}{|c|c|c|c|c|c|c|}
\hline & \multicolumn{2}{|c|}{$M_{0}$} & \multicolumn{2}{|c|}{$M_{0}-0.5$} & \multicolumn{2}{|c|}{$M_{0}+0.5$} \\
\hline & $a$ & $b$ & $\mathrm{a}$ & $\mathrm{b}$ & $a$ & $b$ \\
\hline $1900-1935$ & 5.76 & $0.89 \pm 0.02$ & 5.03 & $0.78 \pm 0.03$ & 6.09 & $0.94 \pm 0.03$ \\
\hline $1936-1972$ & 6.77 & $1.04 \pm 0.02$ & 6.27 & $0.96 \pm 0.02$ & 6.86 & $1.06 \pm 0.02$ \\
\hline $1973-1987.6$ & 5.97 & $0.91 \pm 0.01$ & 5.74 & $0.86 \pm 0.01$ & 6.14 & $0.95 \pm 0.01$ \\
\hline $1987.6-1991.2$ & 5.56 & $0.83 \pm 0.01$ & 5.38 & $0.80 \pm 0.01$ & 5.70 & $0.86 \pm 0.01$ \\
\hline $1991.3-2007$ & 5.64 & $0.85 \pm 0.01$ & 5.51 & $0.82 \pm 0.01$ & 5.73 & $0.87 \pm 0.01$ \\
\hline
\end{tabular}

better seismic hazard map, after applying the MBASS method to determine the threshold magnitude $\left(M_{0}\right)$, then to obtain $b$ values, it will be a profit for mitigating and preventing seismic hazard. We present the following conclusions:

(1) We can obtain a different $b$ value from the determined $M_{0}$ range as shown in Figs. 8 - 12.

(2) The $b$ value of $M_{0}-0.5$ is smaller than the $b$ value of $M_{0}$ +0.5 , and the difference in $b$ value between $M_{0}$ and $M_{0}$ - 0.5 is larger than the difference in $b$ value between $M_{0}$ and $M_{0}+0.5$ (Table 2).

(3) If earthquake events are few and the threshold magnitude is large, such as during $1900-1935$, and $1936-1972$, $M_{0}+0.5$ gives a better result than $M_{0}-0.5$ when using threshold magnitude to calculate $b$ values.
Acknowledgements This study was supported under grant: NSC 97-2745-M-570-001. The authors very appreciate the Central Weather Bureau to support the catalog of Taiwan earthquakes.

\section{REFERENCES}

Aki, K., 1965: Maximum likelihood estimate of $b$ in the formula $\log n=a-b m$ and its confidence limits. Bull. Earthq. Res. Inst., 43, 237-239.

Amorèse, D., 2007: Applying a change-point detection method on frequency-magnitude distributions. Bull. Seismol. Soc. Am., 97, 1742-1749, doi: 10.1785/0120060181. [Link] 
Bender, B., 1983: Maximum likelihood estimation of $b$ values for magnitude-grouped data. Bull. Seismol. Soc. Am., 73, 831-851.

Chen, K. P. and Y. B. Tsai, 2008: A catalog of Taiwan earthquake (1900 - 2006) with homogenized $M_{W}$ magnitudes. Bull. Seismol. Soc. Am., 98, 483-489, doi: 10.1785/0120070136. [Link]

Enescu, B., J. Mori, M. Miyazawa, and Y. Kano, 2009: Omori-Utsu law $c$-values associated with recent moderate earthquakes in Japan. Bull. Seismol. Soc. Am., 99, 884-891, doi: 10.1785/0120080211. [Link]

Frohlich, C. and S. D. Davis, 1993: Teleseismic $b$ value; or, much ado about 1.0. J. Geophys. Res., 98, 631-644, doi: 10.1029/92JB01891. [Link]

Gutenberg, B. and C. F. Richter, 1944: Frequency of earthquakes in California. Bull. Seismol. Soc. Am., 34, 185188.

Ishimoto, M. and K. Iida, 1939: Observations of earthquakes registered with the microseismograph constructed recently. Bull. Earthq. Res. Inst., 17, 443-478.

Kagan, Y. Y., 1999: Universality of the seismic moment-frequency relation. Pure Appl. Geophys., 155, 537-573.

Shi, Y. and B. A. Bolt, 1982: The standard error of the mag- nitude-frequency $b$ value. Bull. Seismol. Soc. Am., 72, 1677-1687.

Utsu, T., 1965: A method for determining the value of $b$ in a formulalog $n=a-b m$ showing the magnitudefrequency relation for earthquake. Geophys. Bull., 13, 99-103. (in Japanese)

Utsu, T., 1992: On seismicity. Report of the Joint Research Institute for Statistical Mathematics. Institute for Statistical Mathematics, Tokyo, 139-157.

Wang, J. H., 1988: $b$ values of shallow earthquakes in Taiwan. Bull. Seismol. Soc. Am., 78, 1243-1254.

Wang, J. H., 1992: Magnitude scales and their relations for Taiwan earthquakes: A review. Terr. Atmos. Ocean. Sci., 3, 449-468.

Wiemer, S., M. Gerstenberger, and E. Hauksson, 2002: Properties of the aftershock sequence of the $1999 M_{W}$ 7.1 Hector Mine earthquake: Implications for aftershock hazard. Bull. Seismol. Soc. Am., 92, 1227-1240, doi: 10.1785/0120000914. [Link]

Wyss, M. and R. Stefansson, 2006, Nucleation points of recent mainshocks in southern Iceland, mapped by b-values. Bull. Seismol. Soc. Am., 96, 599-608, doi: 10.1785/0120040056. [Link] 